\title{
ADVANCED IMAGING MICROSCOPE TOOLS APPLIED TO MICROGRAVITY RESEARCH INVESTIGATIONS
}

\author{
L. Peterson, J. Samson, and D. Conrad \\ NASA Center for Microgravity \\ Automation Technology \\ ERIM \\ PO Box 134001 \\ Ann Arbor, MI 48113-4001 \\ (313) $994-1200$
}

\author{
K. Clark \\ Department of Anatomy and Cell Biology \\ 5805 Medical Science II \\ The University of Michigan Medical School \\ Ann Arbor, MI 48109-0616
}

(313) $763-6225$

\section{Extended Abstract}

The inability to observe and interact with experiments on orbit has been an impediment for both basic research and commercial ventures using the shuttle. In order to open the frontiers of space, the Center for Microgravity Automation Technology has developed a unique and innovative system for conducting experiments at a distance, the "Remote Scientist". The Remote Scientist extends laboratory automation capability to the microgravity environment. While the Remote Scientist conceptually encompasses a broad spectrum of elements and functionalities, the development approach taken is to:

- establish a baseline capability that is both flexible and versatile

- incrementally augment the baseline with additional functions over time

Since last year, the application of the Remote Scientist has changed from protein crystal growth to tissue culture, specifically, the development of skeletal muscle under varying levels of tension. This system includes a series of bioreactor chambers that allow for three-dimensional growth of muscle tissue on a membrane suspended between the two ends of a programmable force transducer that can provide automated or investigator-initiated tension on the developing tissue. A microscope objective mounted on a translation carriage allows for high-resolution microscopy along a large area of the tissue. These images will be mosaiced on orbit to detect features and structures that span multiple images. The use of fluorescence and pseudo-confocal microscopy will maximize the observational capabilities of this system.

A series of ground-based experiments have been performed to validate the bioreactor, the force transducer, the translation carriage and the image acquisition capabilities of the Remote Scientist.

- The bioreactor is capable of sustaining three dimensional tissue culture growth over time.

- The force transducer can be programmed to provide static tension on cells or to simulate either slow or fast growth of underlying tissues in vivo, ranging from $0.2 \mathrm{~mm}$ per day to $32 \mathrm{~mm}$ per day.

- The two-axis translation carriage is capable of scanning the camera along the bioreactor and adjusting the focus with $25 \mu \mathrm{m}$ resolution.

- Time-lapse sequences of images have been acquired, stored and transmitted to a remote computer system.

Although the current application of the Remote Scientist technology is the observation and manipulation of a tissue culture growth system, the hardware has been designed to be easily reconfigured to accommodate a multitude of experiments, including animal observation, combustion studies, protein crystal growth, plant growth and aquatic research. 